\title{
Spinal cord high-grade infiltrating gliomas in adults: clinico- pathological and molecular evaluation
}

\author{
Mohammed Ali Alvi $\mathbb{1}^{1,2} \cdot$ Cristiane M. Ida ${ }^{3}$ Michael A. Paolini ${ }^{3} \cdot$ Panagiotis Kerezoudis $^{1,2}$ - Jenna Meyer ${ }^{4}$. \\ Emily G. Barr Fritcher ${ }^{3}$. Sandy Goncalves ${ }^{1,2}$. Frederic B. Meyer ${ }^{2} \cdot$ Mohammed Bydon $^{1,2} \cdot$ Aditya Raghunathan $^{3}$
}

Received: 17 December 2018 / Revised: 5 March 2019 / Accepted: 6 March 2019 / Published online: 26 April 2019

(c) United States \& Canadian Academy of Pathology 2019

\begin{abstract}
Primary high-grade infiltrating gliomas of the spinal cord are rare, with prior series including limited numbers of cases and reporting poor outcomes. Additionally, the molecular profile of high-grade infiltrating gliomas of the spinal cord has not been well characterized. We identified 13 adult patients whose surgery had been performed at our institution over a 26-yearperiod. Radiologically, nine cases harbored regions of post-contrast enhancement. Existing slides were reviewed, and when sufficient tissue was available, immunohistochemical stains (IDH1-R132H, H3-K27M, H3K27-me3, ATRX, p53 and BRAF-V600E), and a targeted 150-gene neuro-oncology next-generation sequencing panel were performed. The 13 patients included 11 men and 2 women with a median age of 38 years (range $=18-69$ ). Histologically, all were consistent with an infiltrating astrocytoma corresponding to 2016 WHO grades III $(n=5)$ and IV $(n=8)$. By immunohistochemistry, six cases were positive for H3K27M, all showing concomitant loss of H3K27-me3. Next-generation sequencing was successfully performed in ten cases. Next-generation sequencing studies were successfully performed in four of the cases positive for $\mathrm{H} 3 \mathrm{~K} 27 \mathrm{M}$ by immunohistochemistry, and all were confirmed as H3F3A K27M-mutant. Additional recurrent mutations identified included those of TERT promoter $(n=3), T P 53(n=5), \operatorname{PPMID}(n=3), N F 1(n=3), A T R X(n=2)$, and PIK3CA $(n=2)$. No HISTIH3B, HISTIH3C, IDH1, IDH2, or BRAF mutations were detected. Ten patients have died since first surgery, with a median survival of 13 months and 1 year of $46 \%$. Median survival was 48.5 months for H3K27M-positive cases, compared to 1 month for those with TERT promoter mutation and 77 months for those harboring neither $(p=0.019)$. Median survival for cases with TP53 mutations was 11.5 months and for those with PPM1D mutations was 84 months. Our findings suggest that high-grade infiltrating gliomas of the spinal cord in adults represent a heterogeneous group of tumors, with variable outcomes possibly related to their molecular profiles.
\end{abstract}

Previous presentation: An abstract based on this was presented at the 94th Annual Meeting of the American Association of Neuropathologists in June, 2018.

$\triangle$ Aditya Raghunathan

Raghunathan.Aditya@mayo.edu

1 Mayo Clinic Neuro-Informatics Laboratory, Mayo Clinic, Rochester, MN 55902, USA

2 Department of Neurologic Surgery, Mayo Clinic, Rochester, MN 55902, USA

3 Department of Laboratory Medicine \& Pathology, Mayo Clinic, Rochester, MN 55902, USA

4 Medical School for International Health, Faculty of Health Sciences, Ben-Gurion University of the Negev, Be'er Sheva 84105, Israel

\section{Introduction}

High-grade infiltrating gliomas in the spinal cord are relatively rare compared to their intracranial counterparts, accounting for $7.5 \%$ of all intramedullary gliomas and only $1.5 \%$ of all spinal cord tumors $[1,2]$. Only a handful studies on outcomes of high-grade infiltrating gliomas of the spinal cord have been reported in the literature, predominantly as case reports, case series, or as part of a larger cohort of spinal intramedullary tumors [3-8]. However, similar to their supratentorial counterpart, high-grade infiltrating gliomas of the spinal cord have been shown to have an extremely poor prognosis, with reported median survival duration ranging from 12 to 14 months $[4,5]$. Spinal cord surgical procedures typically provide small amounts of tissue even when a resection rather than a biopsy procedure is performed. Owing to this poor prognosis, rare occurrence, 
and limited availability of tumor tissue, high-grade infiltrating glioma of the spinal cord remains incompletely characterized.

The most recent World Health Organization (WHO) Classification of Tumors of the Central Nervous System introduced the new entity of "Diffuse Midline Glioma, H3K27M- mutant" based on histological and molecular characterization, which corresponds to a grade IV designation $[9,10]$. The current understanding regarding this mutation initially came from molecular analysis of midline high-grade gliomas in pediatric and adolescent patients, which revealed a characteristic recurrent lysine to methionine substitution at codon $27(\mathrm{~K} 27 \mathrm{M})$ in histone $\mathrm{H} 3$ variants encoded predominantly by $\mathrm{H} 3.3$ gene $H 3 F 3 A(75-80 \%$ cases) and in H3.1 gene HISTIH3B (25\%). The K27M mutation is located in a critical posttranslational modifiable region of the $\mathrm{N}$-terminal histone tail and seems to act in a dominant-negative manner through inhibition of EZH2 enzymatic subunit of the Polycomb repressive complex 2 (PRC2). H3F3A K27M-mediated PRC2 interaction results in reprogramming of the epigenetic landscape with global reduction of histone $\mathrm{H} 3.3 \mathrm{~K} 27$ methylation, including the trimethylated H3K27 (H3K27-me3). The H3K27-me3 normally serves as a transcriptional repressor; hence, its reduction has been thought to lead to extensive transcriptional modification of the tumor cell regulation, eventually leading to increased expression of cancer-related genes [1120]. These H3K27M-mutant gliomas have been associated with an extremely aggressive behavior, regardless of histologic features in pediatric populations [18-23]; however, within the adult population, the frequency of the mutation and its impact on prognosis is less clear. Within an admixed cohort of 36 pediatric and adult patients with diffusely infiltrating gliomas of the spinal cord, the H3K27M mutation was detected by immunohistochemistry in 16 cases, including 6 of the 11 adults and 10 of the 14 children [24]. A recent study assessing midline gliomas in adults [25] for these common alterations included eight spinal cord gliomas, of which five were found to harbor the H3K27M mutation and none were positive for an IDHI mutation.

Other gene modifications have also been reported associated with H3K27M mutations, including TP53 gene mutations, which have been reported in $50 \%$ of pediatric gliomas $[20,26]$, and in the histone chaperone alpha-thalassemia/mental retardation syndrome $\mathrm{X}$ - linked (ATRX) that affects the loading of histone $\mathrm{H} 3.3$ in heterochromatic telomeric regions [26, 27]. Mutations of $I D H$ and $H 3 F 3 A$ are considered mutually exclusive, demonstrating a potential difference in origin and subsequent behavior of the latter [28]. The telomerase reverse transcriptase gene promoter (TERTp) has been shown to be useful for prognostication, with poor outcomes reported among patients with IDHwild-type infiltrating gliomas [29, 30]. Molecular alterations with potential therapeutic predictive value have been identified, including the BRAF V600E and PPMID mutations [31-33].

In this study, we expand on these findings with a comprehensive molecular analysis of 13 adult patients with high-grade infiltrating glioma of the spinal cord, whose surgery was performed at our institution over a 26-year period - one of the largest single institutional series to date.

\section{Methods}

\section{Patient cohort}

After institutional review board approval, the institutional cancer registry was queried for all patients with high-grade infiltrating glioma of the spinal cord who underwent surgery performed at our institution, identifying 20 cases with tissue specimens available for 19 cases. Of these, 3 patients were aged $<18$ years and thus were excluded. Another 3 patients were excluded because they were found to have supratentorial infiltrating gliomas, and their spinal cord tumors could not be definitely deemed to represent primary tumors. In total, 13 high-grade infiltrating glioma of the spinal cord in adult patients were included for analysis. All cases had research authorization reported. The following variables were reported: (1) patient demographics; (2) presenting symptoms; (3) tumor location; (4) treatment; (5) histological diagnosis; (6) preoperative imaging characteristics, including the presence of post-contrast enhancement and extent of FLAIR signal, if available; (7) presence of hydrocephalus and mass effect; (8) presence of dissemination; (9) followup duration; and (10) vital status at last follow-up.

\section{Immunohistochemistry}

Formalin-fixed, paraffin-embedded tissue sections were stained with hematoxylin and eosin for routine histological review. Immunohistochemical staining was performed on 4$\mu \mathrm{m}$-thick formalin-fixed, paraffin-embedded sections using antibodies against H3K27M (polyclonal, EMD Millipore, Billerica, MA), trimethylated H3K27 (clone C36B11, Cell Signaling Technology, Danvers, MA), IDH1 R132H (clone H09, Dianova, Hamburg, Germany), ATRX (polyclonal, Sigma-Aldrich, St. Louis, MO), and BRAF V600E (clone VE1, Spring Bioscience, Pleasonton, CA).

\section{Molecular testing}

DNA was extracted from 5-micron-thick unstained formalin-fixed, paraffin-embedded tissue sections using the QIAamp ${ }^{\circledR}$ DSP DNA formalin-fixed, paraffin-embedded Tissue Kit (Qiagen Inc., Germantown, MD) with slight 
modifications. Next-generation sequencing library preparation was performed using a QIAseq Targeted DNA custom amplicon-based panel that was designed to interrogate 150 genes associated with central nervous system tumors, including H3F3A, HISTIH3B, HISTIIH3C, IDH1, IDH2, TERT (including promoter region), ATRX, BRAF, CDKN2A, CDKN2B, CDKN2C, CIC, DAXX, EGFR, FGFR1, FGFR2, FGFR3, FUBP1, MYBL1, and MDM2. Libraries were sequenced on an Illumina HiSeq 2500 (Illumina, Inc., San Diego, CA). Sequencing data were processed through a custom bioinformatics pipeline that was developed to detect single-nucleotide polymorphisms and small insertions-deletions ( $<50$ base pairs). Variants at allele frequency of at least $10 \%$ with a minimum of $100 \times$ depth of coverage were evaluated and classified as benign polymorphism, variant of unknown significance, or pathogenic mutation. Only pathogenic mutations were considered for analysis.

\section{Statistical analysis}

Continuous variables have been summarized using mean and standard deviation or median and interquartile ranges and analyzed between patients with and without a mutation using $t$ test or Wilcoxon rank-sum test. Categorical variables have been summarized using frequencies and proportions and compared between patients with and without a mutation using Pearson chi-square or Fischer's exact test.

Kaplan-Meier curves and log-rank test were used to compare survival between patients with and without a mutation.

\section{Results}

\section{Clinical and histological findings}

The 13 patients identified included 11 men and 2 women, with a median age of 38 years (range 18-69 years). The thoracic spinal cord was the most commonly affected region (46\%, $n=6)$ followed by the cervical $(31 \%, n=4)$, thoraco-lumbar $(15 \%, n=2)$, and cervico-thoracic $(8 \%, n$ $=1)$ regions. Radiologically, regions of post-contrast enhancement were observed in 9 cases (Fig. 1a-c). In terms of surgical management, 8 patients underwent a biopsy, 1 patient underwent a gross total resection and 4 patients underwent a subtotal resection.

Histological review of the cases was performed while blinded to the results of immunohistochemical and molecular findings. All cases contained atypical cells with irregular, elongated nuclei arranged in an infiltrative growth pattern characteristic of a diffusely infiltrating astrocytoma.
Applying the histological criteria described in the 2016 WHO Classification of Tumors, 8 of the 13 cases (62\%) showed morphologic features of glioblastoma (WHO grade IV; Fig. 1d) [9]. Five cases showed elevated mitotic activity (3-12 mitoses identified per 10 high-power fields) while lacking definitive microvascular proliferation and necrosis and therefore classified morphologically as anaplastic astrocytoma (WHO grade III).

By immunohistochemistry, six cases were positive for H3K27M (46\%; Fig. 1e), all with concomitant loss of H3K27-me3 expression (Fig. 1. f) and one demonstrating loss of expression of ATRX (the only case in the cohort to show loss of ATRX). All cases were negative for IDH1R132H and BRAF-V600E by immunohistochemistry.

Eleven patients underwent radiotherapy, while eight patients underwent chemotherapy. The most common agent used for chemotherapy was Temozolomide $(n=4)$. Other agents included Cytoxan and Prednisone, CCNU (Lomustine), and Panobinostat (used as off-label), while the agent was unknown in one. One patient also underwent immunotherapy. Only one patient underwent trimodality therapy. The mean follow-up for the cohort was 35 months (range: 0.3-212 months; $\mathrm{SD}=57.6$ ). At last follow-up, 10 out of 13 patients had expired, with a mortality rate of $77 \%$, a median survival of 13 months, and 1-year and 2-year survival rates of $46 \%$ and $50 \%$, respectively.

The clinical and histological data are summarized in Table 1.

\section{Molecular findings}

Next-generation sequencing was successfully performed in 10 (of the 13) cases (specimens obtained within 20002017), with 3 cases yielding insufficient quantity of DNA for analysis due to the small size $\left(<1 \mathrm{~cm}^{2}\right)$ of the available tissue. The results are summarized in Fig. 2. Among these, four of the six cases positive for H3K27M-mutant by immunohistochemistry were found to harbor the $\mathrm{H} 3 \mathrm{~F} 3 \mathrm{~A}$ K27M mutation; the remaining two H3K27M-mutant cases failed sequencing. No HISTIH3B, HISTIH3C, IDHI, $I D H 2, F G F R 1$, or BRAF mutations were detected.

Overall, three distinct molecular groups were apparent: H3K27M-mutant $(n=6), T E R T$ promoter-mutant $(n=3)$, and those lacking mutations in both $(\mathrm{H} 3 \mathrm{~K} 27 \mathrm{M} / T E R T$ promoter wild type; $n=4$ ). In addition to an $H 3 F 3 A$ mutation, all H3K27M-mutant cases frequently showed mutations in TP53, PPM1D, and NF1, as well as a single case each showing mutations in ATRX (in conjunction with an NFI mutation) and in BCOR mutation (in conjunction with a TP53 mutation). All TERT promoter-mutant cases showed the $\mathrm{C} 228 \mathrm{~T}$ mutation. In this group, the only recurrently mutated gene was PIK3CA. A single case also showed TP53, EGFR, and SETD2 mutations in addition to a 

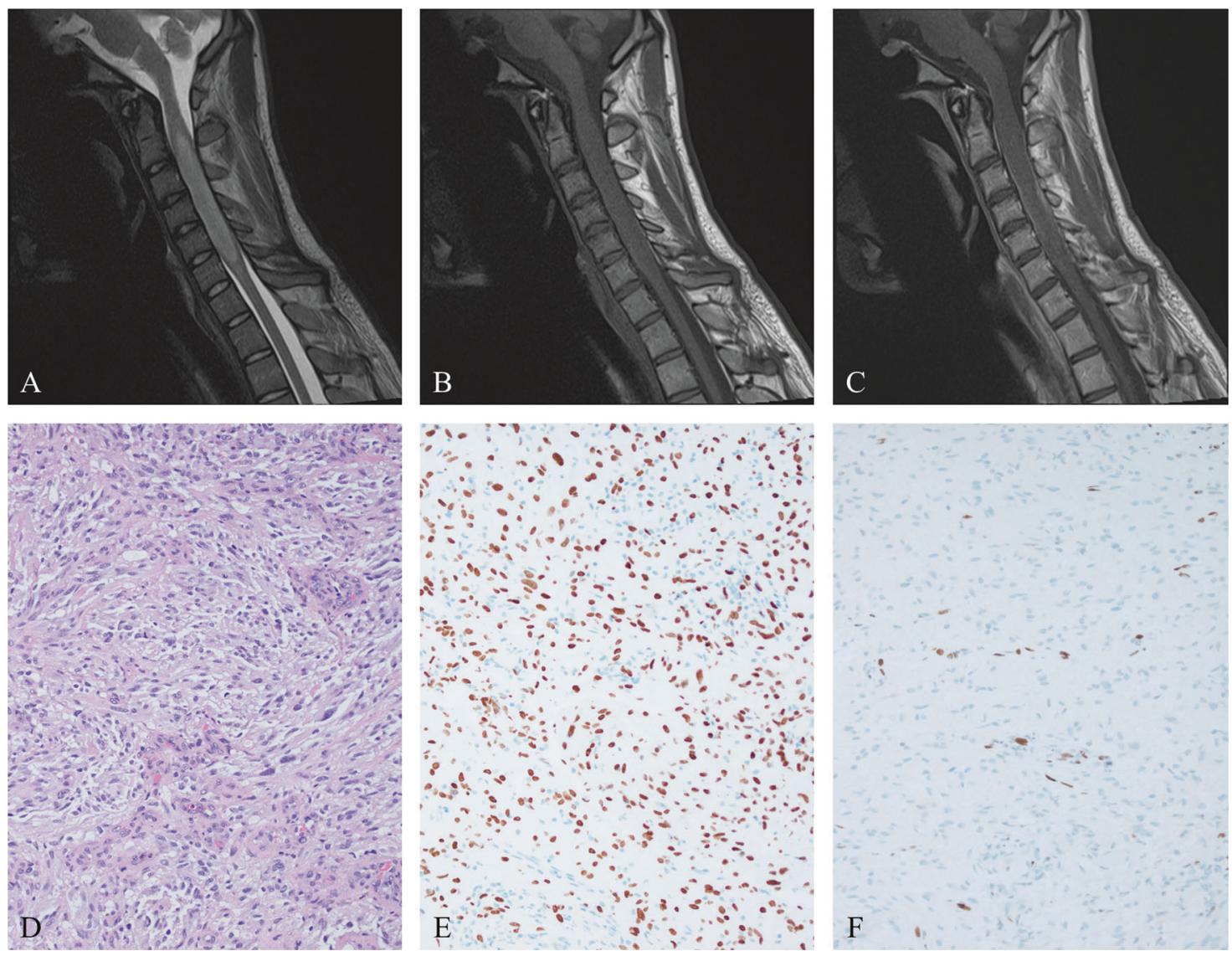

Fig. 1 Illustrative images from case no. 5. a T2-weighted magnetic resonance images (MRIs) reveal an intramedullary mass affecting C2C6 levels. b T1-weighted pre-contrast and $\mathbf{c}$ T1-weighted post-contrast MRIs demonstrate enhancement. d Histological sections show a

cellular, mitotically active infiltrating glioma with microvascular proliferation (hematoxylin and eosin). By immunohistochemistry, e the glioma cells are positive for $\mathrm{H} 3 \mathrm{~K} 27 \mathrm{M}$ and $\mathbf{f}$ show loss of H3K27-me3 expression (all histological images at $\times 200$ magnification)

Table 1 Summary of clinical, radiological and histological features

\begin{tabular}{|c|c|c|c|c|c|c|c|c|c|c|}
\hline Case ID & $\begin{array}{l}\text { Age } \\
\text { (years) }\end{array}$ & Gender & $\begin{array}{l}\text { Spinal } \\
\text { levels } \\
\text { involved }\end{array}$ & $\begin{array}{l}\text { Post-contrast } \\
\text { enhancement on } \\
\text { MRI }\end{array}$ & $\begin{array}{l}\text { Extent of } \\
\text { resection }\end{array}$ & $\begin{array}{l}2016 \\
\text { WHO } \\
\text { grade }\end{array}$ & $\begin{array}{l}\text { Radiation } \\
\text { therapy }\end{array}$ & Chemotherapy (agent) & $\begin{array}{l}\text { Months of } \\
\text { follow-up }\end{array}$ & Status \\
\hline 1 & 69 & $\mathrm{M}$ & $\mathrm{T} 6-\mathrm{T} 7$ & Yes & Biopsy & II & Yes & Yes (Temozolomide) & 77 & Dead \\
\hline 2 & 30 & M & T3-T6, T10 & Yes & Biopsy & III & Yes & Yes (Temozolomide) & 8 & Dead \\
\hline 3 & 38 & M & $\mathrm{T} 4-\mathrm{T} 6$ & NA & $\begin{array}{l}\text { Sub-total } \\
\text { resection }\end{array}$ & IV & Yes & $\begin{array}{l}\text { Yes (Cyclophosphamide } \\
\text { and Prednisone) }\end{array}$ & 212 & Dead \\
\hline 4 & 49 & M & $\mathrm{C} 3-\mathrm{C} 4$ & No & $\begin{array}{l}\text { Gross total } \\
\text { resection }\end{array}$ & III & Yes & None & 8 & Dead \\
\hline 5 & 48 & $\mathrm{~F}$ & $\mathrm{C} 1-\mathrm{C} 2$ & Yes & Biopsy & IV & Yes & None & 0 & Dead \\
\hline 6 & 63 & M & C7-T9 & Yes & Biopsy & III & No & None & 1 & Dead \\
\hline 7 & 27 & M & T11-L1 & NA & $\begin{array}{l}\text { Sub-total } \\
\text { resection }\end{array}$ & IV & Yes & Yes (Lomustine) & 84 & Dead \\
\hline 8 & 52 & $\mathrm{~F}$ & $\mathrm{~T} 3-\mathrm{T} 10$ & NA & Biopsy & III & Yes & None & 4 & Alive \\
\hline 9 & 32 & M & T6-T10 & Yes & Biopsy & III & Yes & Yes (agent unknown) & 0 & Alive \\
\hline 10 & 18 & M & $\mathrm{C} 2-\mathrm{C} 6$ & Yes & $\begin{array}{l}\text { Sub-total } \\
\text { resection }\end{array}$ & III & Yes & Yes (Temozolomide) & 13 & Dead \\
\hline 11 & 36 & M & $\mathrm{C} 1-\mathrm{C} 2$ & Yes & Biopsy & III & Yes & Yes (Temozolomide) & 10 & Dead \\
\hline 12 & 56 & M & $\mathrm{T} 7-\mathrm{L} 2$ & NA & Biopsy & III & Unknown & Unknown & 0 & Dead \\
\hline 13 & 28 & M & T9-T12 & NA & $\begin{array}{l}\text { Sub-total } \\
\text { resection }\end{array}$ & IV & Yes & Yes (Panobinostat) & 15 & Alive \\
\hline
\end{tabular}

$F$ female, $M$ male, $M R I$ magnetic resonance imaging, $N A$ not available, $W H O$ World Health Organization 


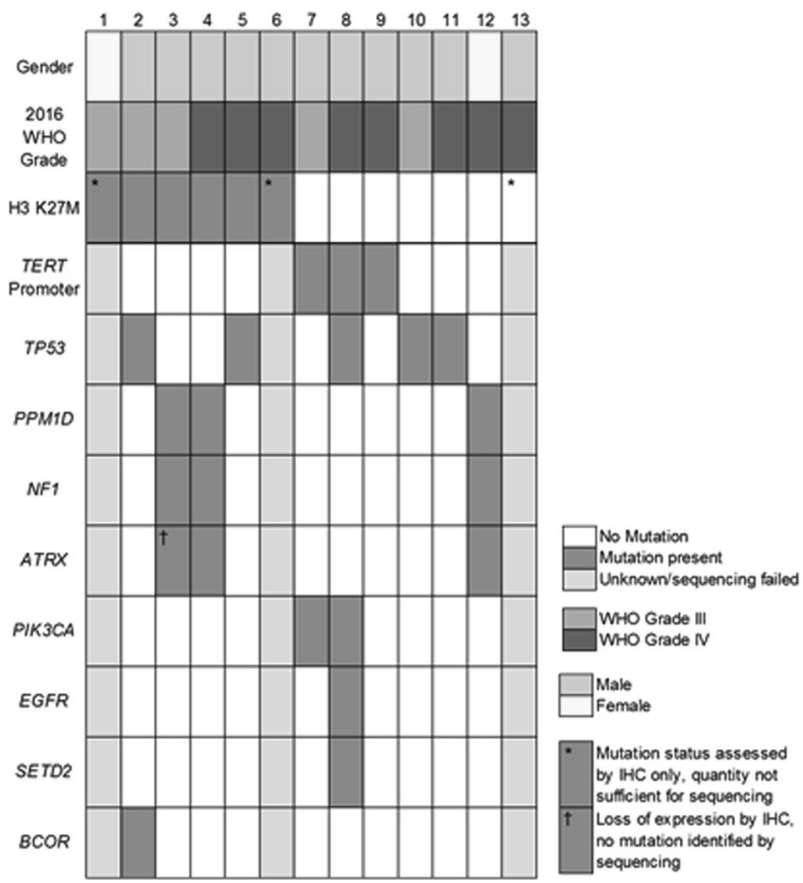

Fig. 2 Oncoprint of mutations identified by 150 -gene next-generation sequencing panel $[38,39]$. Each vertical bar corresponds to a case and all aligned vertical bars across the genes correspond to the same case

PIK3CA mutation. Among the tumors which were H3K27M and TERT promoter wild type, TP53 mutation was the sole abnormality in two cases, while ATRX, NF1 and $P P M 1 D$ mutations co-occurred in one case. These data are summarized in Table 2.

Overall, $P P M 1 D$ mutations were mutually exclusive with TP53 mutations and observed in conjunction with NF1 mutations in all cases. Both PIK3CA mutations occurred in conjunction with a TERT promoter mutation and both $A T R X$ mutations co-occurred with $N F 1$ and PPMID mutations. Of note, the two cases with an ATRX mutation, which were predicted to result in protein truncation in both instances, did not show loss of protein expression by immunohistochemistry (Fig. 2), whereas the single case with loss of ATRX protein expression did not have a detectable sequence alteration by next-generation sequencing and occurred in an H3 K27-mutant tumor (Table 2).

Compared to TERT promoter-mutated tumors, H3K27Mmutant tumors appeared to occur in younger patients and were associated with longer overall survival $(30.5 \pm 9.8$ vs $56 \pm 7, \quad p=0.002 ;$ median survival $=48.5$ months vs 1 month, $p=0.033$, respectively) [Table 3 and Fig. 3]. On pair-wise comparison, no significant difference in the overall survival was found between patients with H3K27Mmutant and H3K27M-wildtype tumors $(p=0.45)$ or between H3K27M-mutant and TERT promoter -mutant tumors $(p=0.13)$. However, patients with TERT promoter mutant high-grade infiltrating gliomas of the spinal cord had significantly lower overall survival compared to those with TERT wild-type tumors-a group that includes all H3K27M-mutant cases $(p=0.01)$.

\section{Discussion}

High-grade gliomas of the spinal cord in adults are rare and carry a poor prognosis $[4,5]$. Although adult diffuse gliomas involving the cerebral hemispheres have undergone extensive molecular characterization, tumors involving the spinal cord have a relative paucity of molecular data available, likely due to their rarity, poor prognosis, and limited availability of tumor tissue. It is not yet well established whether molecular alterations impact prognosis in adult patients with high-grade infiltrating glioma of the spinal cord. The current study is one of the largest reported single-institutional series of high-grade infiltrating glioma of the spinal cord in adults spanning a period of 26 years. While treatment modalities have evolved over this 26-year period, high-grade infiltrating glioma of the spinal cord has remained a clinically aggressive entity, with the median survival of 13 months and 1-year survival of $46.15 \%$ in our cohort.

In our series, we found an incidence of $46.2 \%$ of H3K27M-positive cases $(n=6)$. Two recent series that included pediatric cases reported a rate of $53 \%(n=9 / 17)$ $[21,24]$ among all spinal cord gliomas and $80 \%$ among grade IV gliomas carrying the H3K27M mutation [21, 34]. Picca et al. reported that 5 of the 8 spinal cord gliomas in adults were positive for $\mathrm{H} 3 \mathrm{~K} 27 \mathrm{M}$, while Gessi et al. identified the H3K27M mutation in 6 of the 11 adult spinal cord diffuse gliomas. While, in pediatric cases, diffuse midline gliomas (arising in any location) with the H3K27M mutation carry a worse prognosis compared to wild-type counterparts [35], it is currently uncertain as to what prognostic impact, if any, the presence of this mutation has in spinal cord diffuse gliomas affecting adults. Picca et al., focusing on adult tumors, found no survival difference between H3K27M-mutant vs wild-type tumors when including all midline locations [25]. On the other hand, Yi et al. found that the presence of the H3K27M mutation (detected among 20 of the 25 WHO grade IV gliomas) was associated with longer overall and disease-free survival (40 months) [25, 34]. While this finding was certainly novel, given that almost all studies to date have reported a poor prognosis associated with $\mathrm{H} 3 \mathrm{~K} 27 \mathrm{M}$ mutation, the authors noted that only five cases in their cohort did not have the mutation. Moreover, the treatment characteristics of the patients were not uniform, with only a subset of patients receiving trimodal therapy (surgery, radiation, and Temozolomide) [34]. The median overall survival for H3K27M-positive cases $(n$ $=6$ ) in our cohort was found to be 48 months, compared to 
Table 2 Status of the selected immunohistochemical and molecular markers

\begin{tabular}{llllllll}
\hline Case ID & $H 3 K 27 M$ & H3K27 me3 & IDH1/IDH2 & ATRX & $\begin{array}{l}\text { TERT } \\
\text { promoter }\end{array}$ & $\begin{array}{l}\text { BRAF } \\
\text { V600E }\end{array}$ & Other alteration(s) detected \\
\hline 1 & Negative & Retained & Negative & Retained & Negative & Negative & \\
2 & Positive & Lost & Negative & Retained & Negative & Negative & \\
3 & Negative & Lost & Negative & Retained & Negative & Negative & TP53=p.Arg282Gly, P53=OE-Large \\
4 & Negative & Retained & Negative & Retained & Positive & Negative & PIK3CA=p.Glu542Lys, TP53=p.Arg273His, \\
& & & & & & & EGFR=p.Val774Met, SETD2=p.Asn1392Thrfs*2 \\
5 & Positive & Lost & Negative & Retained & Negative & Negative & \\
7 & Negative & & Negative & Retained & Positive & Negative & PIK3CA=p.Glu545Gln, \\
7 & Positive & Lost & Negative & Lost & Negative & Negative & NF1=p.Lys471Asnfs*2; p.Glu318Lysfs*11, \\
8 & & & & & & PPM1D=p.Glu475Lysfs*8 \\
9 & Negative & Retained & Negative & Retained & Negative & Negative & NF1=p.Asn1683_Thr1690del, PPM1D=p.Trp427X \\
10 & Positive & Lost & Negative & Retained & Negative & Negative & BCOR=p.Arg1163X, TP53=p.Gly245Ser \\
11 & Negative & Retained & Negative & Retained & Negative & Negative & TP53=p.Arg306X \\
12 & Negative & Retained & Negative & Retained & Positive & Negative & \\
13 & Positive & Lost & Negative & Retained & Negative & Negative & NF1=p.Arg385Valfs*2, PPM1D=p.Arg385Valfs*2 \\
\hline
\end{tabular}

Table 3 Summary of the results by molecular subgroup

\begin{tabular}{|c|c|c|c|}
\hline Variable & $\begin{array}{l}\text { H3K27M-mutant ( } n= \\
\text { 6) }\end{array}$ & $\begin{array}{l}\text { TERT promoter mutant } \\
(n=3)\end{array}$ & $\begin{array}{l}\text { No } H 3 F 3 A \text { or TERT } \\
\text { mutation }(n=4)\end{array}$ \\
\hline Age $($ mean $\pm \mathrm{SD})$ & $30.5 \pm 9.8$ & $56 \pm 7$ & $48.8 \pm 15.2$ \\
\hline Females, $n(\%)$ & $1(17 \%)$ & $0(0 \%)$ & $1(25 \%)$ \\
\hline \multicolumn{4}{|l|}{2016 WHO Grade, $n(\%)$} \\
\hline III $(n=5)$ & $3(50 \%)$ & $1(67 \%)$ & $1(25 \%)$ \\
\hline IV $(n=8)$ & $3(50 \%)$ & $2(33 \%)$ & $3(75 \%)$ \\
\hline \multicolumn{4}{|l|}{ Surgical resection, $n(\%)$} \\
\hline Biopsy $(n=8)$ & $3(50 \%)$ & $2(67 \%)$ & $3(75 \%)$ \\
\hline Sub-total resection $(n=4)$ & $3(50 \%)$ & $0(33 \%)$ & $1(25 \%)$ \\
\hline 1) Gross total resection $(n=$ & $0(0 \%)$ & $1(33 \%)$ & 0 \\
\hline \multicolumn{4}{|l|}{ Radiation, $n(\%)$} \\
\hline Yes & $6(100 \%)$ & $1(33 \%)$ & $4(100 \%)$ \\
\hline Unknown & & 1 & \\
\hline \multicolumn{4}{|l|}{ Chemotherapy, $n(\%)$} \\
\hline $\begin{array}{c}\text { Yes }(n=8) \text { (Agent(s) } \\
\text { used } \times \text { number of patients) }\end{array}$ & $\begin{array}{l}\text { Yes }(n=8)(\text { Agent }(s) \\
\text { used } \times \text { number of } \\
\text { patients })\end{array}$ & $\begin{array}{l}\text { Yes }(n=8)(\text { Agent }(s) \\
\text { used } \times \text { number of } \\
\text { patients })\end{array}$ & $\begin{array}{l}\text { Yes }(n=8)(\text { Agent }(s) \\
\text { used } \times \text { number of } \\
\text { patients })\end{array}$ \\
\hline Unknown & 1 & 1 & 0 \\
\hline $\begin{array}{l}\text { Follow-up in months, mean } \pm \\
\text { SD }\end{array}$ & $24 \pm 30.4$ & $3 \pm 4.35$ & $75.7 \pm 96.6$ \\
\hline Died, $n(\%)$ & $4(67 \%)$ & $3(100 \%)$ & $3(75 \%)$ \\
\hline
\end{tabular}

WHO World Health Organization
77 months in tumors without the mutation $(n=4)$, which are in contrast to those reported by Yi et al., who reported a longer survival among those with H3K27M mutations [34].

Among adult diffuse gliomas, TERT promoter mutation occurring in the absence of IDH mutations and $1 \mathrm{p} / 19 \mathrm{q}$ - codeletion is associated with poor prognosis [21]. In the current series, we identified 3 (of the 13) cases harboring a TERT promoter mutation. Among this group, the median survival was found to be just 1 month, as compared to 75.7 months in tumors without an $\mathrm{H} 3 \mathrm{~K} 27 \mathrm{M} / T E R T$ promoter 

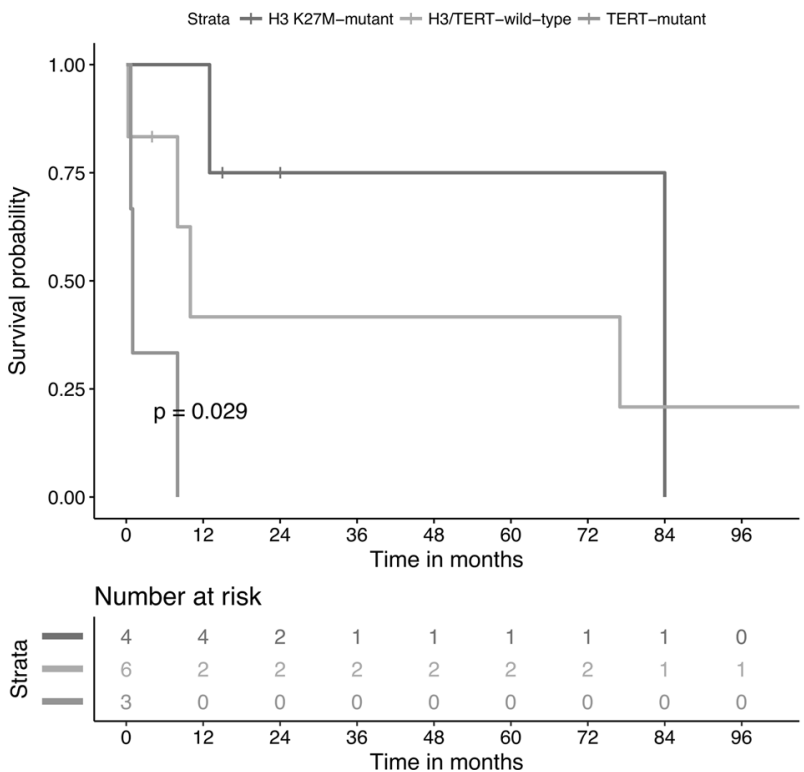

Fig. 3 Kaplan-Meier survival analysis by H3K27M and TERT promoter mutation status

mutation. Picca et al. showed that TERT promoter mutations are also associated with shorter survival. In their cohort, TERT promoter mutations are significantly enriched among H3K27M/IDH-wild-type adult midline gliomas, with concurrent TERT promoter mutation and $\mathrm{H} 3 \mathrm{~K} 27 \mathrm{M}$ mutation in 2 (of the 8) spinal cord gliomas, and in overall 4 (of the 116) adult midline gliomas from various sites [25]. In our cohort, all three TERT promoter mutant cases were negative for H3K27M. The significance of concurrent H3K27M and TERT promoter mutation among midline gliomas, and spinal cord gliomas in particular, remains to be determined. While data regarding the presence of additional alterations is unavailable in the report by Picca et al., we have been able to identify alterations occurring in the presence and absence of the H3K27M mutation (Table 2), which may help provide some insight into the molecular landscape present in these rare tumors.

All three $P P M 1 D$ mutations identified in this study were novel truncating alterations within the regulatory C-terminal domain, which is a mutational hotspot region in human cancer [36]. As previously described in brainstem infiltrating gliomas [33], PPMID mutations were mutually exclusive with TP53 mutations in our series of adult spinal cord high-grade gliomas. In contrast to brainstem infiltrating gliomas, wherein PPM1D mutations were only observed in H3K27M-mutant tumors, PPM1D mutations were seen in both H3K27M-mutant and H3/IDH/TERT wild-type cases in our series. It was also noteworthy that the ATRX status by immunohistochemistry and sequencing did not correlate in this study: the two cases with an ATRX truncating mutation did not show loss of protein expression by immunohistochemistry, whereas the single case with ATRX loss by immunohistochemistry did not have an ATRX sequence alteration detectable by next-generation sequencing. While immunohistochemical loss of ATRX expression in the absence of a detectable ATRX mutation has been observed [37], preserved ATRX protein expression in the presence of an ATRX truncating mutation is somewhat unusual. The identified ATRX mutations occurred within exons 26 and 30 (out of 35 total exons) and seem to have escaped nonsensemediated decay; the expressed mutant proteins, however, are likely defective as at least one of them lacks the helicase functional domain.

Our findings suggest that high-grade infiltrating glioma of the spinal cord of adult patients are a heterogeneous group of tumors, with main molecular groups including H3K27M-mutant, TERT promoter-mutant, and those harboring neither. Among adult high-grade infiltrating gliomas of the spinal cord, tumors with either an $\mathrm{H} 3 \mathrm{~K} 27 \mathrm{M}$ or a TERT promoter mutation appear to represent clinically and prognostically distinct groups, with H3K27M-mutant tumors appearing to affect younger patients and being associated with longer overall survival when compared to TERT promoter-mutant tumors. Similar to the findings of Picca et al. pertaining to supratentorial midline tumors among adults, IDH-wildtype/H3K27M-wild-type infiltrating gliomas seems to delineate a group of clinically aggressive tumors, and this group frequently shows TERT promoter mutation in the spinal cord.

In summary, we have characterized 13 high-grade infiltrating gliomas affecting the spinal cord in adult patients. Although the spinal cord is midline in location, these tumors appear to be share similar molecular profiles as diffuse gliomas affecting midline and non-midline locations at other sites. Additionally, the differences in molecular profiles may also have prognostic significance for these patients. Given the small size of our study cohort, evaluation of clinico-pathological and molecular features in larger multi-institutional cohorts may help confirm the prognostic significance of this molecular heterogeneity. Further, prospective studies may help determine whether adult patients with high-grade infiltrating glioma of the spinal cord would indeed benefit from targeted therapies in the similar manner as molecularly defined gliomas affecting other sites.

\section{Compliance with ethical standards}

Conflict of interest The authors declare that they have no conflict of interest.

Publisher's note: Springer Nature remains neutral with regard to jurisdictional claims in published maps and institutional affiliations. 


\section{References}

1. Bowers DC, Weprin BE. Intramedullary spinal cord tumors. Curr Treat Options Neurol. 2003;5:207-12.

2. Seki T, Hida K, Yano S, et al. Surgical outcomes of high-grade spinal cord gliomas. Asian Spine J. 2015;9:935-41.

3. Shen C-X, Wu J-F, Zhao W, et al. Primary spinal glioblastoma multiforme: a case report and review of the literature. Medicine. 2017;96:e6634.

4. Iwata K, Nakagawa H, Hashizume Y. Significance of MIB-1, PCNA indices, and p53 protein over-expression in intramedullary tumors of the spinal cord. Noshuyo Byori. 1996;13:73-8.

5. Strik HM, Effenberger $O$, Schäfer $O$, et al. A case of spinal glioblastoma multiforme: immunohistochemical study and review of the literature. J Neurooncol. 2000;50:239-43.

6. Ciappetta P, Salvati M, Capoccia G, et al. Spinal glioblastomas: report of seven cases and review of the literature. Neurosurgery. 1991;28:302-6.

7. Raco A, Esposito V, Lenzi J, et al. Long-term follow-up of intramedullary spinal cord tumors: a series of 202 cases. Neurosurgery. 2005;56:972-81. Discussion 972-81

8. Miller DC. Surgical pathology of intramedullary spinal cord neoplasms. J Neurooncol. 2000;47:189-94.

9. Louis DN, Perry A, Reifenberger G, et al. The2016 World Health Organization Classification of Tumors of the Central Nervous System: a summary. Acta Neuropathol. 2016;131: 803-20.

10. Tabouret E, Nguyen AT, Dehais C, et al. Prognostic impact of the 2016 WHO classification of diffuse gliomas in the French POLA cohort. Acta Neuropathol. 2016;132:625-34.

11. Bender S, Tang Y, Lindroth AM, et al. Reduced H3K27me3 and DNA hypomethylation are major drivers of gene expression in K27M mutant pediatric high-grade gliomas. Cancer Cell. 2013;24:660-72.

12. Chan K-M, Fang D, Gan H, et al. The histone H3.3K27M mutation in pediatric glioma reprograms $\mathrm{H} 3 \mathrm{~K} 27$ methylation and gene expression. Genes Dev. 2013;27:985-90.

13. Lewis PW, Müller MM, Koletsky MS, et al. Inhibition of PRC2 activity by a gain-of-function $\mathrm{H} 3$ mutation found in pediatric glioblastoma. Science. 2013;340:857-61.

14. Chan KM, Han J, Fang D, et al. A lesson learned from the H3. $3 \mathrm{~K} 27 \mathrm{M}$ mutation found in pediatric glioma: a new approach to the study of the function of histone modifications in vivo? Cell Cycle. 2013;12:2546-52.

15. Lulla RR, Saratsis AM, Hashizume R. Mutations in chromatin machinery and pediatric high-grade glioma. Sci Adv. 2016;2: e1501354.

16. Daoud EV, Rajaram V, Cai C, et al. Adult brainstem gliomas with H3K27M mutation: radiology, pathology, and prognosis. J Neuropathol Exp Neurol. 2018;77:302-11.

17. Buczkowicz P, Hoeman C, Rakopoulos P, et al. Genomic analysis of diffuse intrinsic pontine gliomas identifies three molecular subgroups and recurrent activating ACVR1 mutations. Nat Genet. 2014;46:451-6.

18. Chiang JC, Ellison DW. Molecular pathology of paediatric central nervous system tumours. J Pathol. 2017;241:159-72.

19. Gielen GH, Gessi M, Hammes J, et al. H3F3A K27M mutation in pediatric CNS tumors: a marker for diffuse high-grade astrocytomas. Am J Clin Pathol. 2013;139:345-9.

20. Wu G, Broniscer A, McEachron TA, et al. Somatic histone H3 alterations in pediatric diffuse intrinsic pontine gliomas and nonbrainstem glioblastomas. Nat Genet. 2012;44:251-3.
21. Solomon DA, Wood MD, Tihan T, et al. Diffuse midline gliomas with histone H3-K27M mutation: a series of 47 cases assessing the spectrum of morphologic variation and associated genetic alterations. Brain Pathol. 2016;26:569-80.

22. Khuong-Quang DA, Buczkowicz P, Rakopoulos P. K27M mutation in histone H3.3 defines clinically and biologically distinct subgroups of pediatric diffuse intrinsic pontine gliomas. Acta Neuropathol. 2012;124:439-47.

23. Brandner S, von Deimling A. Diagnostic, prognostic and predictive relevance of molecular markers in gliomas. Neuropathol Appl Neurobiol. 2015;41:694-720.

24. Gielen GH, Dreschmann V, Waha A, et al. High frequency of $\mathrm{H} 3 \mathrm{~F} 3 \mathrm{AK} 27 \mathrm{M}$ mutations characterizes pediatric and adult high-grade gliomas of the spinal cord. Acta Neuropathol. 2015;130:435-7.

25. Picca A, Berzero G, Bielle F, et al. FGFR1 actionable mutations, molecular specificities, and outcome of adult midline gliomas. Neurology. 2018;90:e2086-94.

26. Schwartzentruber J, Korshunov A, Liu X-Y, et al. Driver mutations in histone H3.3 and chromatin remodelling genes in paediatric glioblastoma. Nature. 2012;482:226-31.

27. Szenker E, Ray-Gallet D, Almouzni G. The double face of the histone variant H3.3. Cell Res. 2011;21:421-34.

28. Sturm D, Witt H, Hovestadt V, et al. Hotspot mutations in H3F3A and IDH1 define distinct epigenetic and biological subgroups of glioblastoma. Cancer Cell. 2012;22:425-37.

29. Chen W-J, He D-S, Tang R-X, et al. Ki-67 is a valuable prognostic factor in gliomas: evidence from a systematic review and meta-analysis. Asian Pac J Cancer Prev. 2015;16:411-20.

30. Lee Y, Koh J, Kim S-I, et al. The frequency and prognostic effect of TERT promoter mutation in diffuse gliomas. Acta Neuropathol Commun. 2017;5:62.

31. Chi AS, Batchelor TT, Yang D, et al. BRAF V600E mutation identifies a subset of low-grade diffusely infiltrating gliomas in adults. J Clin Oncol. 2013;31:e233-6.

32. Takahashi Y, Akahane T, Sawada T, et al. Adult classical glioblastoma with a BRAF V600E mutation. World J Surg Oncol. 2015;13:100.

33. Zhang L, Chen LH, Wan $\mathrm{H}$, et al. Exome sequencing identifies somatic gain-of-function PPM1D mutations in brainstem gliomas. Nat Genet. 2014;46:726-30.

34. Yi S, Choi S, Shin DA, et al. Impact of H3.3 K27M mutation on prognosis and survival of grade IV spinal cord glioma on the basis of new 2016 World Health Organization Classification of the central nervous system. Neurosurgery. 2018. https://doi.org/10. 1093/neuros/nyy150

35. Karremann M, Gielen GH, Hoffmann M, et al. Diffuse high-grade gliomas with $\mathrm{H} 3 \mathrm{~K} 27 \mathrm{M}$ mutations carry a dismal prognosis independent of tumor location. Neuro Oncol. 2018;20:123-31.

36. Dudgeon C, Shreeram S, Tanoue K, et al. Genetic variants and mutations of PPM1D control the response to DNA damage. Cell Cycle. 2013;12:2656-64.

37. Jiao Y, Killela PJ, Reitman ZJ, et al. Frequent ATRX, CIC, FUBP1 and IDH1 mutations refine the classification of malignant gliomas. Oncotarget. 2012;3:709-22.

38. Cerami E, Gao J, Dogrusoz U, Gross BE, Sumer SO, Aksoy BA, et al. The cBio Cancer Genomics Portal: An Open Platform for Exploring Multidimensional Cancer Genomics Data. Cancer Discovery. 2012;2:401-4.

39. Gao J, Aksoy BA, Dogrusoz U, Dresdner G, Gross B, Sumer SO, et al. Integrative Analysis of Complex Cancer Genomics and Clinical Profiles Using the cBioPortal. Science Signaling 2013;6. 